\title{
Selective Removal, Recovery, and Characterization of Immunoglobulins from Human Colostrum ${ }^{1}$
}

\author{
T. WILlIAM HUTCHENS, J, SCOTT MAGNUSON, AND TAI-TUNG YIP \\ USDA/ARS Children's Nutrition Research Center, Department of Pediatrics, Baylor College of Medicine and \\ Texas Children's Hospital. Houston. Texas 77030
}

\begin{abstract}
Investigations into the mechanisms by which Ig in human colostrum influence the development and maturation of both the gastrointestinal and the immune systems of human milk-fed term and preterm infants have been restricted by the paucity of purified human milk Ig. We have developed a simple adsorption procedure for the selective removal and quantitative recovery $(95-100 \%)$ of intact Ig (secretory IgA, IgG, and IgM) present in human colostral whey. The procedure exploits the rapid, ionicstrength dependent, thiophilic adsorption of Ig during a single pass of colostral whey through a column of beaded agarose with immobilized thioether-sulfone ligands (Anal Biochem 1986;159:217-226). The purity and composition of the adsorbed Ig were verified by SDS-PAGE and sensitive silver-staining protein detection procedures; proteins of approximately $78-80 \mathrm{kD}$ (secretory component), 50-60 kD (heavy chain), and $25 \mathrm{kD}$ (light chain) were observed. The identity, structural integrity, and relative concentrations of the recovered Ig were confirmed by high-performance size-exclusion chromatography, Ouchterlony immunodiffusion, rocket immunoelectrophoresis and ELISA. These results were analyzed and compared with reported values for the concentration of human milk Ig. Thus, the use of thiophilic adsorption appears to facilitate 1) selective removal of Ig from colostrum, enabling the evaluation of remaining components for growth- and immune-potentiating properties, and 2) selective immobilization and recovery of Ig from colostrum under conditions consistent with preserved bjologic activity. (Pediatr Res 26: 623-628, 1989)
\end{abstract}

\section{Abbreviations}

SIgA, secretory IgA

HEPES, N-2-hydroxyethyl piperizine-N'-2-ethanesulfonic acid

HPSEC, high-performance size-exclusion chromatography

Several factors present in human colostrum provide for increased host defense mechanisms in the breast-fed infant $(1-10)$.

Received May 26, 1989; accepted August I, 1989.

Comespondence T. William Hutchens, Department of Pediatrics, Children's Nutrition Research Center, Baylor College of Medicine, 1100 Bates Street, Houston, TX 77030 .

Supported in part with federal funds from the U.S. Department of Agriculture, Agricultural Research Service under Cooperative Agreement no. 58-7MN1-6-100.

'The contents of this publication do not necessarily reflect the views or policies of the U.S. Department of Agriculture, nor does mention of trade names, $\infty \mathrm{m}$ mercial products, or organizations imply endorsement by the U.S. Government.
Substantial quantities of Ig are found in both colostrum and mature milk, although the major Ig in human colostrum is $\operatorname{SIgA}$ (11-13). Various structural features unique to the sIgA dimer (11.4 S, $420 \mathrm{kD}$ ) collectively make it resistant to the low $\mathrm{pH}$ and degratory proteolytic environment of the gastrointestinal tract (14-16). On the basis of these properties, sIgA functions as an efficient Ig for the transmission of passive immunity to breastfed infants.

The protective effects of $\operatorname{sIgA}$ at the intestinal mucosa are becoming well established $(1,5,9,17-21)$; the magnitude and mechanism(s) by which this particular Ig benefits the human milk-fed infant remain the subject of intense investigations (2227). Indeed, uncertainties surround the metabolic fate of undigested sIgA $(25,26)$ and its potential immune modulating properties. IgA fractions obtained from adult human sera (no secretory component) have been examined for the prevention of necrotizing enterocolitis in preterm infants (19). However, slgA remains the stated $\mathrm{Ig}$ of choice for these and similar investigations $(17,19,28-30)$

Because of difficulties associated with the isolation of intact slgA $(14,15)$, it has been impossible to date to obtain sufficient quantities to evaluate the ability of SIgA alone to promote the passive immunity of term and preterm infants who are denied fresh human milk. Indeed, significant quantities of sIgA dimer are required for both in vitro and in vivo investigations. Thus, a simple and efficient method for the preparative scale isolation of intact $\operatorname{SIg} A$ dimer is needed.

Several methods are available for the purification of $\mathrm{Ig}$ in general. Most of these methods were developed for the isolation of gammaglobulins using the preparative separation techniques then available. Such methods as the Cohn fractionation procedure (31), ammonium sulfate precipitation, hydroxyapatite adsorption, and the combination of ion-exchange and size-exclusion chromatography (e.g., Ref. 32) are adequate, but labor intensive and produce lower than desired recoveries. More importantly, for the selective removal of Ig from human milk, these methods are not sufficiently selective to allow the recovery of Igdepleted colostral whey. Conversely, some very selective biospecific affinity methods, such as immobilized lectins (33), immobilized protein $A(34)$, and immobilized protein $G$ (35) columns, although invaluable for certain uses, are too restrictive in the classes of Ig they adsorb, are expensive, and require harsh con. ditions for removal of the adsorbed $\operatorname{Ig}(35,36)$.

We have developed a separation technique to enable both reutilization of the Ig-free milk products and the subsequent desorption of purified antibodies using mild conditions consistent with preserved antibody function. Thiophilic adsorption on sulfone-thioether agarose has been used to remove Ig from serum and to purify MAb (37-41). A high degree of selectivity and good recovery of antibody activity were demonstrated $(38,39)$. In this report we show the thiophilic adsorption method to be equally effective in selectively removing all classes of Ig found in human. 
colostrum, enabling for the first time, not only further studies of the biologic significance of ingested $\operatorname{sIgA}$, but also that of Igdepleted human colostrum.

\section{MATERIALS AND METHODS}

Synthesis of T-gel for Ig adsorption. The method for synthesis of divinylsulfone cross-linked agarose and neutralization of reactive groups with $\beta$-mercaptoethanol was as described by Porath and coworkers $(37,42)$. A total of $100 \mathrm{~g}$ of suction-dried Sepharose 6B (Pharmacia Fine Chemicals, Piscataway, NJ) was suspended in $100 \mathrm{~mL}$ of $0.5 \mathrm{M}$ sodium carbonate, $\mathrm{pH}$ 11.0. Divinyl sulfone (Fluka, Ronkonkoma, NY) was added $(5 \mathrm{~mL})$ and the mixture was shaken for $20 \mathrm{~h}$ at room temperature $\left(21^{\circ} \mathrm{C}\right)$ on a rotary shaker. The gel was washed thoroughly with distilled water and suction-dried. The gel $(70 \mathrm{~g})$ was suspended in $70 \mathrm{~mL}$ of 0.1 $M$ sodium bicarbonate ( $\mathrm{pH} 9.0$ ) and $7 \mathrm{~mL}$ of mercaptoethanol (Fluka) was added for incubation overnight at room temperature on a rotary shaker. Excess mercaptoethanol was washed away with water and the gel was stored semidry (suction-dried) at $4^{\circ} \mathrm{C}$. The immobilized ligand density on the completed $\mathrm{T}$-gel was estimated by elemental ( $C, S, N)$ analyses before and after each step in the synthesis procedure (Galbraith Laboratories, Knoxville, TN).

Preparation of human colostral whey. Samples of both fresh and frozen colostrum were supplied by the USDA/ARS Children's Nutrition Research Center Milk Bank, Department of Pediatrics, Baylor College of Medicine, Houston, TX. The colostrum was collected from each breast on $\mathrm{d} 2$ to 4 postpartum with a mechanical pump and stored at $-80^{\circ} \mathrm{C}$. Immediately before use, frozen colostrum was thawed at $37^{\circ} \mathrm{C}$, adjusted to $\mathrm{pH} 4.33$, and incubated for $1 \mathrm{~h}$ at $4^{\circ} \mathrm{C}$. The casein and fat were then removed by centrifuging the low $\mathrm{pH}$ colostrum at $45,000 \mathrm{rpm}$ for $1 \mathrm{~h}$ at $4^{\circ} \mathrm{C}$ in a Beckman SW 60 rotor (Beckman Instruments, Fullerton, $\mathrm{CA}$ ). The fatty layer was removed and the whey was decanted.

Thiophilic adsorption and recovery of purified Ig. Ammonium sulfate $(10 \% \mathrm{w} / \mathrm{v})$ and $0.5 \mathrm{M}$ sodium chloride were added to the whey sample and the $\mathrm{pH}$ was adjusted to 8.0. Chromatographic details for analytical and semipreparative scale isolation procedures are given in the figure legends. The T-gel columns were equilibrated with $20 \mathrm{mM}$ HEPES buffer $(\mathrm{pH} 8.0)$ containing $10 \%$ or $12.5 \%(\mathrm{w} / \mathrm{v})$ ammonium sulfate and $0.5 \mathrm{M} \mathrm{NaCl}$. After sample application, the columns were washed overnight with column equilibration buffer. Elution of adsorbed Ig was achieved by removal of ammonium sulfate from the column elution buffer. Finally, the columns were eluted with $50 \%$ ethylene glycol in $20 \mathrm{mM}$ HEPES. Fractions of $2 \mathrm{~mL}$ (analytical) or $6 \mathrm{~mL}$ (semipreparative) were collected and the UV absorbance at 280 nm was recorded (Beckman DU-70 analytical spectrophotometer, Beckman Instruments).

$S D S-P A G E$. The purity and mol wt of the isolated Ig subunits were examined by electrophoresis using a 10 to $20 \% \mathrm{~T}, 3 \% \mathrm{C}$ gradient gel under denaturing conditions at a constant current of $40 \mathrm{~mA}$ per gel slab (43). Before denaturation and electrophoresis, samples were dialyzed against $20 \mathrm{mM}$ HEPES buffer $(\mathrm{pH}$ 8.0 ) in dialysis tubing with a mol wt cutoff of 3500 . The gel was silver-stained using a modification of the basic procedure described by Morrissey (44).

High-performance size-exclusion chromatography. Protein $\mathrm{M}_{\mathrm{r}}$ calibrators and samples of the purified Ig were analyzed on a TSK 4000SW column $(7.5 \times 600 \mathrm{~mm}$, Phenomenex, Torrance, CA) at $4^{\circ} \mathrm{C}$ using a Beckman System Gold HPLC system (Beckman Instruments) equipped with a model 166 or 167 UV detector. The column eluent was $20 \mathrm{mM}$ sodium phosphate buffer (pH 6.8) containing $0.4 \mathrm{M} \mathrm{KCl}$. A flow rate of $0.5 \mathrm{~mL} / \mathrm{min}$ was used and protein elution was monitored by UV absorbance at $280 \mathrm{~nm}$.

Immunochemical characterization of purified $\mathrm{Ig}$. The isolated human colostral whey Ig were identified using Ouchterlony immunodiffusion (45) and rocket immunoelectrophoresis (46). Polyclonal antibodies directed against $\gamma, \alpha$, and $\mu$ heavy chain determinants (Dako Corporation, Santa Barbara, CA) were used for identification of $\mathrm{Ig}$ class. The gels were stained with $\mathrm{CoO}-$ massie blue.

Quantilative determination of $1 g$ concentrations. Ig concentrations and recoveries were determined using ELISA. Polyclonal antibodies directed against $\gamma, \alpha$, and $\mu$ heavy chain determinants (Dako Corporation) were immobilized on a plastic 96-well microtiter plate. The antigen was first incubated with the primary, immobilized antibody. The plates were then washed before incubation with a second antibody conjugated to horseradish peroxidase. The color reaction was developed with hydrogen peroxide in citrate buffer containing o-phenylenediamine (Sigma Chemical Co., St. Louis, MO) and read at $490 \mathrm{~nm}$.

\section{RESULTS}

Selective removal of Ig from human colostral whey. Using 10 or $12.5 \%(\mathrm{w} / \mathrm{v})$ ammonium sulfate in the column equilibration buffer, the majority of proteins in human colostrum did not adsorb to the $T$-gel stationary phase, but instead were eluted unretained in the flow-through fractions (Fig. 1, peak $A$ ). Some proteins (mainly lactoferrin) were slightly retained when the $12.5 \%$ ammonium sulfate column equilibration buffer was used, and were located in the trailing edge of the flow-through peak. After an extensive column wash period $(15 \mathrm{~h})$, all the weakly interacting proteins were removed. Thereafter, removal of ammonium sulfate from the column elution buffer resulted in the elution of the adsorbed proteins (Ig) in a sharp peak (Fig. 1, peak $B$ ). Any remaining bound Ig not eluted with the HEPES buffer were recovered completely with $50 \%$ ethylene glycol (Fig. 1, peak $C)$ as previously described for serum $\operatorname{Ig}(38)$. Although not shown, 10 to $30 \%$ ethylene glycol may be included with the $20 \mathrm{mM}$ HEPES, $0.5 \mathrm{M} \mathrm{NaCl}$ elution buffer to achieve a more quantitative elution of sIgA from the T-gel in a single step. The significance of sIgA elution with and without ethylene glycol is not currently understood.

Realizing the importance of removing immunoglobulins from larger volumes of colostrum, we endeavored to increase the scale of the isolation procedure to show the effectiveness of the T-gel with larger volumes of colostral whey sample. It can also be seen in Figure 1 that the manner in which the colostral whey proteins were eluted on a larger (semipreparative) scale is the same as that observed with the analytical scale procedure. Ig were eluted with the HEPES buffer at neutral $\mathrm{pH}$ with no detectable decrease in purity or selectivity. This procedure has been applied successfully to the selective adsorption of Ig from porcine colostral whey using $1 \mathrm{-kg}$ columns of $\mathrm{T}$-gel to process $1 \mathrm{~L}$ of whey per day (Hutchens TW, Magnuson IS, and Yip T.T, unpublished data).

Determination of purity and structural characterization of purified Ig. The identity and structural integrity of those proteins selectively adsorbed to the T-gel (Fig. 1, peak $B$ ) were analyzed by high-performance size-exclusion chromatography (HPSEC) to evaluate molecular size properties under nondenaturing conditions. Two major protein peaks were observed eluting from the TSK4000SW HPSEC column (Fig. 2). IgM was present only in low concentrations and was not resolved from the much larger quantities of sIgA. The first major peak of protein (peak D) observed by HPSEC eluted in exactly the same position as standard secretory IgA (dimer). The second protein peak (peak $E$ ) eluted as a pure protein in the position equivalent to secretory component $(78,000 \mathrm{D})$. The identities of these two protein peaks as eluted from the HPSEC column were verified as free secretory component (peak $E$ ), and sIgA dimer (peak D), respectively, by Ouchterlony immunodiffusion (Fig. 4) and rocket immunoelectrophoresis (Fig. 5).

Analyses of both the adsorbed Ig and the unretained colostral whey proteins from the $T$-gel column were routinely performed using SDS-PAGE with silver-staining (Fig. 3). The combination 